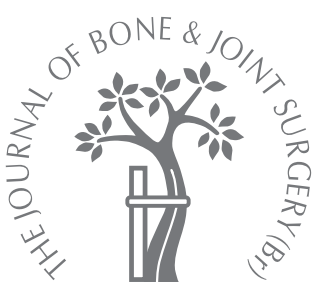

A. Cil, C. J. H. Veillette, J. Sanchez-Sotelo, J. W. Sperling, C. Schleck, R. H. Cofield

From the Mayo Clinic, Rochester, USA

\footnotetext{
A. Cil, MD, Orthopaedic Surgeon

C. J. H. Veillette, MD, Orthopaedic Surgeon J. Sanchez-Sotelo, MD, Orthopaedic Surgeon J. W. Sperling, MD, MBA, Orthopaedic Surgeon C. Schleck, BS, Biostatistics R. H. Cofield, MD, Orthopaedic Surgeon Department of Orthopaedics Mayo Clinic, 200 First Street SW, Rochester, Minnesota 55905, USA

Correspondence should be sent to $\mathrm{Dr}$ R. H. Cofield; e-mail: cofield.robert@mayo.edu

(C)2009 British Editorial Society of Bone and Joint Surgery doi:10.1302/0301-620X.91B1. $21094 \$ 2.00$

$J$ Bone Joint Surg $[\mathrm{Br}]$ 2009;91-B:75-81. Received 1 April 2008; Accepted after revision 21 August 2008
}

\title{
Revision of the humeral component for aseptic loosening in arthroplasty of the shoulder
}

\begin{abstract}
Between 1976 and 2004, 38 revision arthroplasties (35 patients) were performed for aseptic loosening of the humeral component. The mean interval from primary arthroplasty to revision was 7.1 years ( 0.4 to 16.6). A total of 35 shoulders (32 patients) were available for review at a mean follow-up of seven years (2 to 19.3).

Pre-operatively, 34 patients $(97 \%)$ had moderate or severe pain; at final follow-up, 29 $(83 \%)$ had no or only mild pain $(p<0.0001)$. The mean active abduction improved from $88^{\circ}$ to $107^{\circ}(p<0.01)$; and the mean external rotation from $37^{\circ}$ to $46^{\circ}(p=0.27)$. Excellent or satisfactory results were achieved in 25 patients $(71 \%)$ according to the modified Neer rating system. Humeral components were cemented in 29, with ingrowth implants used in nine cases. There were 19 of standard length and 17 were longer (two were custom replacements and are not included). Bone grafting was required for defects in $\mathbf{1 1}$ humeri. Only two glenoid components were left unrevised. Intra-operative complications included cement extrusion in eight cases, fracture of the shaft of the humerus is two and of the tuberosity in four. There were four re-operations, one for recurrent humeral loosening, with $89 \%$ survival free of reoperations at ten years.
\end{abstract}

Revision surgery for aseptic loosening of the humeral component provides reliable pain relief and modest improvement of movement, although there is a substantial risk of intraoperative complications. Revision to a total shoulder replacement gives better results than to a hemiarthroplasty.

The increase in the number of shoulder arthroplasties performed raises concern that a higher number of revision procedures may be inevitable. Failure of a shoulder arthroplasty can result from soft-tissue problems, bony deficiencies, infection, and component wear or loosening. ${ }^{1}$ Loosening of the humeral component is much less common than with the glenoid component. ${ }^{2-6}$ Analysis of the different stem designs and fixation techniques has revealed that although radiological changes around humeral components are common, especially for the press-fit implants, the clinical outcome does not appear to be dependent on the presence of these radiolucencies. ${ }^{7-10}$ Despite these radiological changes around the stems, loosening is rarely reported as a primary cause for revision surgery. ${ }^{7-13}$ Some authors have reported no radiolucent lines around the humeral component.

Revision of loose humeral components is uncommon, and there are few publications on the outcome. The purpose of this study was to evaluate the results, complications and survival after revision of loose humeral components, with or without associated revision or removal of the glenoid element.

\section{Patients and Methods}

Between 1976 and 2004, 290 revisions of humeral components were performed in our institution for reasons such as infection, rotator cuff tear, loosening, peri-prosthetic fracture and to enable exposure during revision of the glenoid component. Of these, 44 shoulders had revision of the humeral component for loosening confirmed at the time of revision. For inclusion in this study, patients had to have a complete pre-operative evaluation, operative records, a minimum of two years' clinical follow-up, and a complete set of radiographs available for review, including the pre-operative, immediate post-operative and most recent obtained at least one year after revision. Six patients had less than one year of radiological follow-up, although they had a minimum of two years of clinical review. These patients were included in the survival analysis, but were otherwise excluded from further assessment. Three patients died during the two-year 




Fig. 1a

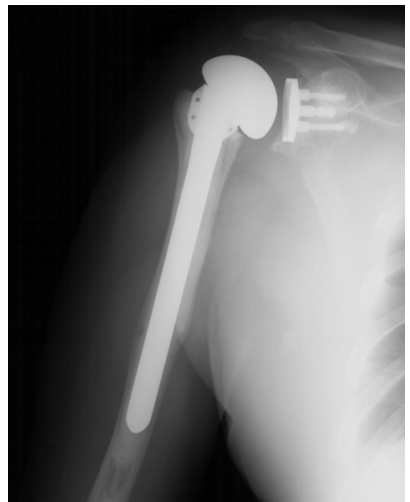

Fig. 1b
Pre-operative anteroposterior radiograph showing a) a 65-year-old man with humeral osteolysis, a loosened and shifted ingrowth humeral component implanted seven years previously, worn glenoid polyethylene and wearing of the posterior aspect of metal glenoid tray and b) radiographs at the time of surgery showing that the glenoid component was revised and a long-stemmed humeral component was cemented in place.

follow-up from causes unrelated to surgery. The pre- and early post-operative data obtained from these patients were analysed, but were not included in the final follow-up analysis.

Thus, 38 shoulders in 35 patients were included in the review, and 35 shoulders in 32 patients were available for analysis at the final follow-up. Of the 38 procedures, 19 were performed on men, and 19 on women. The mean age at the time of revision was 63 years (30 to 85 ). Of the procedures, 22 involved the right and 16 the left shoulder. The primary diagnosis was osteoarthritis in 21, post-traumatic arthritis in eight, rheumatoid arthritis in six, arthritis due to instability in two and cuff tear arthropathy in one shoulder. The mean number of previous operations was 1.7 (1 to 5 ). There were 25 primary arthroplasties performed at our institution; 13 had been undertaken elsewhere and referred for revision. The mean interval between the primary arthroplasty and the revision was 7.1 years $(0.4$ to 16.6). The mean follow-up from revision was 6.9 years $(2$ to 19.3 ).

Of the 38 shoulder arthroplasties, one was a replacement of the humeral head and the rest were total shoulder replacements (TSRs). There were 19 Neer components (3M Company, St Paul, Minnesota), 17 Cofield components (Smith-Nephew, Memphis, Tennessee), one Global component (DePuy, Warsaw, Indiana) and one Aequelis component (Tornier, Stafford, Texas). A total of 11 humeral components were cemented, 13 were press-fitted and 14 were ingrowth implants. The glenoid components had been cemented in 21 cases (one hemiarthroplasty did not require a glenoid component) and ingrowth glenoid components were used in 16 shoulders.

Operative data. At revision, a deltopectoral approach was used in 21 cases and an anteromedial exposure in $17 .{ }^{14}$
There was no problem with the healing of the deltoid in any of these patients.

All of the humeral components were loose. An anterior cortical window was necessary for removal of one pressfitted humeral component, which was rotationally unstable with no vertical instability. At revision, 26 rotator cuffs were found to be intact, eight were attenuated, and there were five had full-thickness tears, all of which were repaired. There were three nonunions and two malunions of the tuberosities. There were deficiencies of the tuberosities in two shoulders. The underlying diagnosis in the patients with tuberosity problems was post-traumatic arthritis in four cases, inflammatory arthritis in two cases and osteoarthritis in one. The erythrocyte sedimentation rate, white blood cell count and C-reactive protein levels were normal in all patients. Histological examination by frozen section was available for all shoulders. All but one showed no evidence of acute inflammation; the one patient with acute inflammatory changes had negative tissue cultures. However, four other shoulders had positive intraoperative tissue cultures, with Propionibacterium acnes in two cases and coagulase-negative Staphylococcus in two cases, despite a lack of clinical or histological evidence of infection. Loosening of the humeral component was not thought to be due to infection in these cases, so they were not excluded from the study.

Glenoid bone loss was graded intra-operatively on the basis of location and severity, as previously described. ${ }^{14,15}$ Based on location, the defects were categorised as central (17 shoulders) or combined (11 shoulders), with no cases of isolated peripheral bone loss. Based on severity, the deficiencies were classified as mild in six shoulders, moderate in seven and severe in 15. In 25 cases the glenoid component and surrounding bone cement were removed and TSR converted to a humeral head replacement because it was felt that there was inadequate bone stock for secure fixation of the glenoid. The resulting defect was grafted with freshfrozen cancellous allograft chips in 21 cases. In ten cases the glenoid components were revised, and they remained as TSRs. In two others the glenoid components were not revised, as there was no polyethylene wear or loosening. The single humeral head replacement had erosion of the glenoid cartilage without bone deficiency, and was converted to a TSR.

The humeral components used at revision were Neer implants (3M Company) in four shoulders and Cofield (Smith-Nephew) in 32 shoulders. In 11 cases, contained defects in the bone stock of the proximal humerus were grafted with fresh-frozen cancellous allograft chips. Severe, uncontained proximal humeral bone loss required a custom proximal humeral replacement in two cases. Of the humeral components used during revision, 19 were standard in length $(<150 \mathrm{~mm})$ and 17 were intermediate or long (> $150 \mathrm{~mm}) ; 29$ of the humeral components were cemented and nine with ingrowth surfaces were press-fitted (Fig. 1). 
Grading of the clinical outcome. All patients who have an arthroplasty performed at our institution are prospectively followed in our Total Joint Registry. Standard clinical data are recorded pre-operatively and at each follow-up visit. Patients who are unable to return for follow-up are contacted and answer our validated shoulder questionnaire, which records pain, function, activities of daily living, range-of-movement and satisfaction. ${ }^{16}$ Pain is graded as 1 (no pain), 2 (slight pain), 3 (pain after unusual activity), 4 (moderate pain) or 5 (severe pain). Range of movement is recorded in degrees for active abduction and active external rotation, and for internal rotation is measured based on the ability of the thumb to reach posterior vertebral segments. The results were classified according to a modified Neer rating system ${ }^{17,18}$ as excellent (no or slight pain, active abduction to $\geq 140^{\circ}$, external rotation to $\geq 45^{\circ}$ and patient satisfaction), satisfactory (no or slight pain or moderate pain only with vigorous activities, active elevation to $\geq 90^{\circ}$, external rotation to $\geq 20^{\circ}$ and satisfaction), and unsatisfactory (none of the above, or re-operation).

Radiological analysis. Three projections were used for radiological analysis: a $40^{\circ}$ posterior oblique radiograph with external rotation of the humerus, a $40^{\circ}$ posterior oblique radiograph with internal rotation of the humerus, and an axillary view. When available, fluoroscopically-positioned spot views were also evaluated. Glenohumeral subluxation was assessed with regard to direction, and graded as none, mild (centre of prosthetic head translated $<25 \%$ relative to the centre of the glenoid component), moderate (centre of the prosthetic head translated $25 \%$ to $50 \%$ relative to the centre of the glenoid), or severe (centre of the prosthetic head translated $>50 \%$ relative to the centre of the glenoid). Peri-prosthetic lucency was graded as 0 (none), 1 (1 mm incomplete), 2 (1 mm complete), 3 (1.5 mm incomplete), 4 (1.5 mm complete), or 5 ( $\geq 2 \mathrm{~mm}$ complete) for both components. The humeral component-bone interface for uncemented components and humeral component-bone-cement interface for cemented components were divided into eight zones as previously described. ${ }^{8,9}$ Radiolucent lines were evaluated according to their presence or absence, zonal location and thickness. The three observers compared the early post-operative and final radiographs to judge whether either component had changed position. This was considered to have occurred when two or three of the three observers noted medial migration, subsidence, tilting or a combination of these..$^{7-9}$

The maximum width of lucent lines surrounding the components, whether the lines were complete or incomplete, and the assessment for change in position were tabulated. A glenoid component was defined to be radiologically at risk for loosening if two or three of the observers identified medial migration or tilt, or if there was a complete radiolucent line, some part of which was $\geq 1.5 \mathrm{~mm}$ wide. A humeral component was identified to be radiologically at risk if two or three of the three observers identified tilt or subsidence of the component, or if there was a radiolucent line $\geq 2 \mathrm{~mm}$ wide in three or more zones. ${ }^{7-9}$
Statistical analysis. The association between risk factors and outcomes was assessed using Fisher's exact test (discrete) or Wilcoxon's rank sum test (continuous), as appropriate. The associations of the clinical and functional outcomes with gender, rotator cuff status, humeral component fixation, hemiarthroplasty versus TSR and subluxation grade were assessed with two-sample $t$-tests. Comparisons of pre-operative with post-operative status for pain, active forward elevation and external and internal rotation were made using a paired $t$-test. An analysis of variance was used to test for differences in pain, active abduction and external and internal rotation among the patients with three different Neer grades. Survival free of any revision/re-operation was estimated using the KaplanMeier survival method, with revision estimated as a function of time since the index revision surgery. Estimates were reported with $95 \%$ confidence intervals (CI). The $\alpha$ level was set at 0.05 for statistical significance.

\section{Results}

Complications and revisions. There were 14 intra-operative complications in 11 patients occurring either during removal of the humeral component and/or cement, or during preparation of the humeral canal and insertion of the revision component. Cement extrusion occurred in eight shoulders. Three were explored and the cement removed, either because of proximity to the radial nerve (two cases) or because the cement lay within a diaphyseal intraoperative fracture (one case). In one of the three the defect was grafted with cancellous allograft. There were six intraoperative fractures (16\%). Two were undisplaced spiral diaphyseal fractures and were not internally fixed. There were four tuberosity fractures, three involving both tuberosities. These were grafted and fixed with sutures at the time of revision. One patient had an undisplaced of the fracture greater tuberosity that was not fixed.

During follow-up four patients had a further operation. One who had rheumatoid arthritis developed aseptic loosening of the revised humeral component at seven months. Four years later she had a peri-prosthetic fracture requiring revision of the humeral component. One patient with a hemiarthroplasty complained of pain in the shoulder, and underwent an operation elsewhere five years after the revision. One patient with a metal-backed glenoid component had wear of the glenoid polyethylene diagnosed 12 years after the revision and underwent exchange of the polyethylene (Fig. 1). One patient who had cuff tear arthropathy developed a sinus tract on the anterior aspect of the shoulder within three weeks of the revision. Cultures grew coagulase-negative Staphylococcus. The infection could not be controlled and the patient underwent excision arthroplasty.

The estimated survival rate for revision shoulder arthroplasty for humeral component aseptic loosening free of reoperation was $98 \%$ (95\% CI, 93 to 100) at one year, $89 \%$ $(95 \% \mathrm{CI}, 79$ to 100$)$ at five years, and $89 \%$ (95\% CI, 79 to 




Fig. 2a

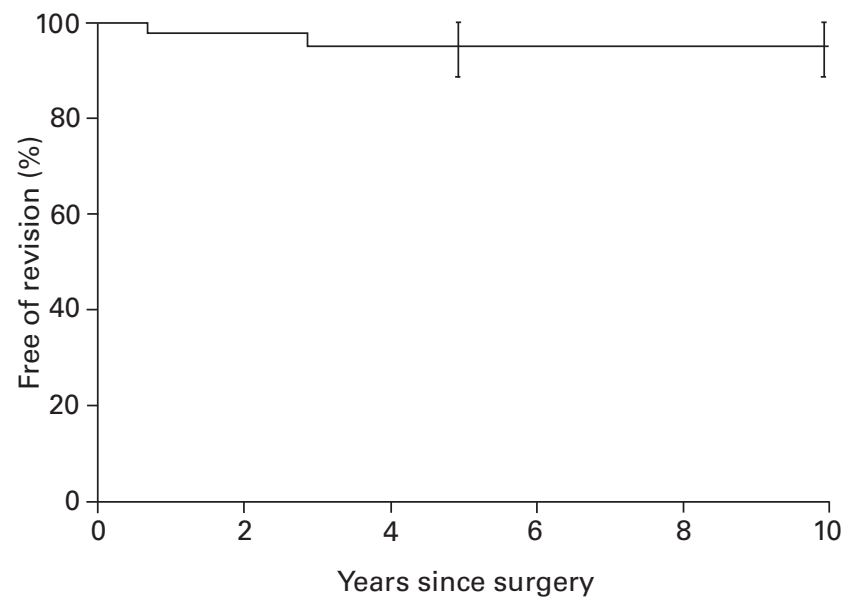

Fig. $2 b$

Graphs showing a) overall survival rates free of re-operation and b) free of revision of the humeral component with $95 \%$ confidence intervals.

\begin{tabular}{|c|c|c|c|}
\hline & \multicolumn{3}{|c|}{ Construct after revision operation ${ }^{*}$} \\
\hline & HA $(n=22)$ & TSR $(n=13)$ & p-value \\
\hline Pain score & 2.8 & 1.9 & 0.01 \\
\hline Active abduction $\left({ }^{\circ}\right)$ & 93 & 130 & 0.004 \\
\hline External rotation $\left({ }^{\circ}\right)$ & 43 & 50 & 0.43 \\
\hline Internal rotation & L5 & L4 & 0.19 \\
\hline \multicolumn{4}{|l|}{ Neer result rating (number) } \\
\hline Excellent or satisfactory & 13 & 12 & \\
\hline Unsatisfactory & 9 & 1 & 0.03 \\
\hline \multicolumn{4}{|l|}{ Subjective rating (number) } \\
\hline Much better or worse & 15 & 13 & \\
\hline Same or worse & 7 & 0 & 0.001 \\
\hline
\end{tabular}

$100)$ at ten years. The estimated survival rate free of humeral revision/removal was $98 \%$ (95\% CI, 93 to 100) at one year, $95 \%$ (95\% CI, 88 to 100 ) at five years, and $95 \%$ (95\% CI, 88 to 100 ) at ten years (Fig. 2).

Four shoulders with positive intra-operative tissue cultures were seen by the orthopaedic infectious disease service. Two of these positive intra-operative cultures were thought to be due to contamination. They were not treated, and the patients did not develop signs of infection during the follow-up intervals of 25 and 27 months, respectively. The other two patients received a limited course of antibiotic treatment without further surgery and there were no signs of infection at a follow-up of three years and 15 years, respectively.

Pain. Pain scores decreased from a mean of 4.2 to 2.5 $(\mathrm{p}<0.01)$. A total of 34 of 35 patients $(97 \%)$ had moderate or severe pain prior to revision, and 29 patients $(83 \%)$ had no or mild pain at the most recent follow-up. At the final follow-up, TSR resulted in better pain relief than hemiarthroplasty, with mean pain scores of 1.9 and 2.8, respectively $(\mathrm{p}<0.01)$ (Table I). Improvement in pain was not associated with gender, the presence of a rotator cuff tear at the time of revision, the type of humeral component used, fixation of the humeral component, subluxation grade or radiolucency (humeral or glenoid) at the time of most recent follow-up ( $p>0.1)$.

Range of movement. Active abduction improved from a mean of $88^{\circ}\left(30^{\circ}\right.$ to $\left.160^{\circ}\right)$ to $107^{\circ}\left(10^{\circ}\right.$ to $\left.180^{\circ}\right)$ ( $\left.\mathrm{p}<0.01\right)$. TSR resulted in a better active abduction than hemiarthroplasty, with mean active elevation of $130^{\circ}\left(80^{\circ}\right.$ to $\left.180^{\circ}\right)$ and $93^{\circ}\left(10^{\circ}\right.$ to $\left.165^{\circ}\right)$, respectively $(\mathrm{p}<0.01)$ (Table I). External rotation improved from a mean of $39^{\circ}\left(-10^{\circ}\right.$ to $\left.70^{\circ}\right)$ to $46^{\circ}$ $\left(0^{\circ}\right.$ to $\left.90^{\circ}\right)$, but did not reach statistical significance $(\mathrm{p}>0.1)$. The mean range of internal rotation stayed the 


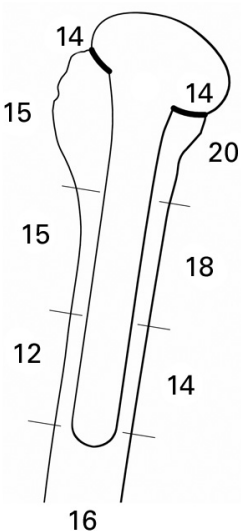

Fig. 3a



Fig. 3b
Drawing showing the zonal distribution and sizes of the peri-prosthetic lucencies among the humeral components. The numbers refer to the number of components having lucency in each zone. Figure $3 \mathrm{a}$ - Radiolucent lines of $\geq 2 \mathrm{~mm}$ pre-operatively. Figure $3 \mathrm{~b}-$ Radiolucent lines $\geq 2 \mathrm{~mm}$ at final follow-up.

same, with the ability of the thumb to reach the fifth lumbar vertebra $(p>0.1)$. The pre-operative to post-operative changes in movement did not vary with respect to gender, the presence of a rotator cuff tear at the time of revision, type of humeral component used, fixation of the humeral component, or subluxation grade at the most recent follow-up ( $\mathrm{p}>0.1$ ).

Radiographs. Pre-operatively, $28(74 \%)$ of the humeral components (Fig. 3) and 23 (61\%) of the glenoid components were found to be at risk. Immediately after revision, none of the humeral components and only one glenoid component was identified to be at risk of loosening. At a mean radiological follow-up of 5.1 years (1 to 16.4 ), four $(11 \%)$ humeral components and five $(13 \%)$ glenoid components were identified to be at risk. None of the glenoid components and one humeral component changed position during the follow-up period. No humeral radiolucent lines were observed in 22 replacements $(63 \%)$. The remaining cases had radiolucent lines limited to one zone in three shoulders, two zones in four shoulders, three zones in two shoulders, four zones in one shoulder, six zones in one shoulder, and eight zones in two shoulders. The thickness of the radiolucent lines was $>2 \mathrm{~mm}$ in four cases. Three of these were cemented, and one was an ingrowth humeral component. These four cases were the ones which were identified as at risk for loosening. In three of these cases the length of the humeral component was less than $150 \mathrm{~mm}$ (standard humeral component). The estimated survival rate for radiological humeral loosening at final follow-up was 97\% (95\% CI, 91.5 to 100 ) at one year, 90.6\% (95\% CI, 78 to 100$)$ at five years, and $90.6 \%(95 \%$ CI, 78 to 100$)$ at ten years.

Pre-operatively, 11 shoulders $(29 \%)$ had moderate to severe subluxation, anteriorly in two, superiorly in six, and posteriorly in three shoulders. At the time of most recent follow-up, 13 (34\%) had moderate to severe subluxation, nine in a superior direction and four in a posterior direction. Seven of these patients were subluxating pre-operatively. However, six patients developed subluxation following their revision surgery. There was no significant association between subluxation at the most recent followup and humeral head replacement versus TSR or rotator cuff tear at the time of revision surgery $(p>0.1)$. At the most recent follow-up, glenoid bone erosion was present in 18 shoulders $(47 \%)$ which was mild in two, moderate in seven and severe in nine.

Satisfaction and result rating. Overall, 15 patients (43\%) stated that they were much better compared with their preoperative condition, 13 patients $(37 \%)$ were better, four patients $(11 \%)$ were the same, and three patients $(9 \%)$ were worse. When the seven dissatisfied patients were compared with the patients who were satisfied, pain and active abduction were found to be significantly different $(\mathrm{p}<0.01)$. There was no significant association between unsatisfactory results and rotator cuff tear, glenoid erosion, subluxation or humeral radiolucency $(\mathrm{p}>0.1)$. There was a significant association between dissatisfaction and having humeral head replacement versus TSR at revision procedure $(\mathrm{p}<0.01)$.

When the modified Neer rating system was applied, five shoulders $(13 \%)$ had excellent, $20(53 \%)$ had satisfactory and ten $(26 \%)$ the unsatisfactory results were examined in detail, three had a re-operation, seven had a limited range of movement, five were not satisfied with the procedure, and five had moderate to severe pain. There were no significant associations between Neer rating and rotator cuff tear, glenoid erosion or subluxation at the time of most recent follow-up ( $\mathrm{p}>0.1$ ). However, there was a significant association between Neer rating and humeral radiolucency at follow-up $(\mathrm{p}<0.05)$. Three of four patients with humeral components at risk for loosening had unsatisfactory Neer ratings. There was also a significant association between humeral head replacement versus TSR and Neer rating $(\mathrm{p}<0.05)$. Nine of ten unsatisfactory Neer ratings were in patients who had a hemiarthroplasty.

\section{Discussion}

The initial implant designed by Neer for replacement of the humeral head was press-fitted. ${ }^{19}$ The Neer II implant was designed for cemented use. ${ }^{20}$ Detailed radiological analysis of 72 press-fit Neer II humeral components with a mean follow-up of 4.1 years showed that over $55 \%$ were judged to be at risk for clinical loosening. ${ }^{7}$ Of 37 shoulder arthroplasties, using either the press-fitted Neer II prosthesis or the modular shoulder prosthesis (3M Company, St Paul, Minnesota), 59\% had radiolucency around the humeral component and $19 \%$ were judged to be at risk for loosening after a mean of 9.2 years. ${ }^{10}$ In contrast, the use of a tapered press-fit metaphyseal stem in 127 shoulders showed radiolucency in $61 \%$ after a mean of three years, 
with the majority of the lucencies being at the tip of the component. None were considered to be at risk for loosening. ${ }^{12}$ Similarly, a study of 43 cemented Neer II humeral components with a mean of 6.6 years of radiological follow-up revealed only one $(2 \%)$ to be at risk for loosening, despite $37 \%$ of the components having incomplete radiolucent lines. ${ }^{8}$ Tissue ingrowth components have been designed to reduce loosening, yet radiological analysis demonstrated a rate of $9.7 \%$ of the Cofield 1 ingrowth humeral components at risk of loosening with a mean of 4.6 years follow-up. ${ }^{9}$ Despite the frequency of radiological changes around the humeral components, mechanical failure due to loosening has not been a common cause for revision surgery, ${ }^{2}$ even after a minimum of 15 years of follow-up in young patients. ${ }^{21}$ Except for anecdotal case reports ${ }^{11,22}$ there are no series analysing the clinical and radiological outcome of revision of loose humeral components. To our knowledge, the present study is the only one reporting those outcomes, and therefore several technical and clinical points merit additional discussion.

In approximately one-third of the patients revision was associated with an intra-operative complication, most commonly extrusion of cement from the perforated humeral shaft, and/or fracture of the humerus. Thus, an intra-operative radiograph during the procedure is very helpful. However, these complications, when treated adequately either during the operation or by altering physiotherapy in the early post-operative period, did not result in an adverse outcome in any of the patients.

Osteolysis of the proximal humerus and tuberosities required cancellous bone grafting in approximately onethird of patients. Except for one, none of these patients at the final follow-up had humeral components at risk of radiological loosening, and in none was there evidence of proximal humeral osteolysis. Thus, bone grafting can effectively treat contained proximal humeral bone deficiencies. However, extensive proximal humeral deficiency might preclude reconstruction using standard off-the-shelf humeral components and bone grafting. In these circumstances custom-made components may be necessary, and were used in the present series in two patients. Pre-operative planning is essential in order to have the necessary implants and grafts available during revision procedures.

In three-quarters of the cases cement was used for fixation of the humeral component at revision. Our routine practice is to use ingrowth components whenever press-fit of the proximal part of the humeral component is possible, in both primary and revision cases. However, in most revisions, proximal humeral osteolysis and endosteal bone erosion preclude a successful press-fit. Filling the endosteal defects with cement, and in some cases particulate bone graft, added security to the fixation of the humeral component, and therefore cement is advocated whenever a tight press-fit cannot be achieved proximally and at the isthmus during revision surgery.
There was significant relief of pain after revision with $83 \%$ of the patients having no pain or only mild pain after follow-up of approximately seven years. The mean active abduction also improved significantly after revision from $88^{\circ}$ to $107^{\circ}$. However, improvement of pain and active abduction was significantly better in the patients who had TSR than hemiarthroplasty. Subjectively, none of the patients in the TSR group were dissatisfied, and nine of ten unsatisfactory Neer results were in patients with a hemiarthroplasty. Consequently, where bone stock permits, every effort should be made either to keep the glenoid component or revise it to ensure improved and more predictable pain relief.

There was a high rate of instability of the shoulder following revision. More than one-third of the patients had moderate to severe subluxation radiologically. With the number of patients in the study we could not identify any risk factors for the development of instability. However, this finding did not measurably affect the clinical outcome in terms of pain relief, range-of-movement or satisfaction.

As a result, with estimated survival free of re-operation due to any cause of $89 \%$, and free of humeral radiolucency of $90.6 \%$ at ten years, revision arthroplasty for loosening of the humeral component, with or without associated glenoid component revision or removal, is a successful procedure. Despite substantial intra-operative complications and post-operative instability it can reliably provide pain relief and improve function, especially when the revision procedure is a TSR.

No benefits in any form have been received or will be received from a commercial party related directly or indirectly to the subject of this article.

\section{References}

1. Petersen SA, Hawkins RJ. Revision of failed total shoulder arthroplasty. Orthop Clin North Am 1998;29:519-33.

2. Chin PYK, Sperling JW, Cofield RH, et al. Complications of total shoulder arthroplasty: are they fewer or different? J Shoulder Elbow Surg 2006;15:19-22.

3. Cofield RH, Chang W, Sperling JW. Complications of shoulder arthroplasty. In: lanotti JP, Williams GR Jr, eds. Disorders of the shoulder: diagnosis and management Philadelphia: Lippincott, Williams \& Wilkins, 1999:571-93.

4. Cofield RH. Revision procedures for shoulder arthroplasty. In: Morrey BF, ed. Recon structive surgery of the joints. Second ed. New York: Churchill Livingstone, 1996:789-99.

5. Boileau P, Sinnerton RJ, Chuinard C, Walch G. Arthroplasty of the shoulder. $J$ Bone Joint Surg [Br] 2006;88-B:562-75.

6. Haines JF, Trail IA, Nuttall D, Birch A, Barrow A. The results of arthroplasty in osteoarthritis of the shoulder. J Bone Joint Surg [Br] 2006;88-B:496-501.

7. Sanchez-Sotelo J, Wright TW, O'Driscoll SW, Cofield RH, Rowland CM. Radiographic assessment of uncemented humeral components in total shoulder arthroplasty. J Arthroplasty 2000;16:180-7.

8. Sanchez-Sotelo J, O'Driscoll SW, Torchia ME, Cofield RH, Rowland CM. Radiographic assessment of cemented humeral components in shoulder arthroplasty. J Shoulder Elbow Surg 2001;10:526-31.

9. Sperling JW, Cofield RH, O'Driscoll SW, Torchia ME, Rowland CM. Radiographic assessment of ingrowth total shoulder arthroplasty. J Shoulder Elbow Surg 2000:9:507-13

10. Verborg 0, El-Abiad R, Gazielly DF. Long term results of uncemented humeral components in shoulder arthroplasty. J Shoulder Elbow Surg 2007;16:13-18.

11. Klimkiewicz JJ, lannotti JP, Rubash HE, et al. Aseptic loosening of the humeral component in total shoulder arthroplasty. J Shoulder Elbow Surg 1998;7:422-6. 
12. Matsen FA III, lanotti JP, Rockwood CA Jr. Humeral fixation by press-fitting of a tapered metaphyseal stem: a prospective radiographic study. J Bone Joint Surg [Am] 2003;85-A:304-8.

13. Raiss $\mathbf{P}$, Aldinger PR, Kasten $\mathbf{P}$, Rickert $\mathbf{M}$, Loew $\mathbf{M}$. Total shoulder replacement in young and middle-aged patients with glenohumeral osteoarthritis. J Bone Joint Surg [Br] 2008;90-B:764-9.

14. Gill DRJ, Cofield RH, Rowland C. The anteromedial approach for shoulder arthroplasty: the importance of the anterior deltoid. J Shoulder Elbow Surg 2004;13:532-7.

15. Antuna SA, Sperling JW, Cofield RH, Rowland CM. Glenoid revision surgery after total shoulder arthroplasty. J Shoulder Elbow Surg 2001;10:217-24.

16. Smith AM, Barnes SA, Sperling JW, et al. Patient and physician-assessed shoulder function after arthroplasty. J Bone Joint Surg [Am] 2006;88-A:508-13.

17. Cofield RH. Total shoulder arthroplasty with the Neer prosthesis. J Bone Joint Surg [Am] 1984;66-A:899-906.
18. Neer CS, Watson KC, Stanton FJ. Recent experience in total shoulder replacement. J Bone Joint Surg [Am] 1982;64-A:319-37.

19. Neer CS II. Articular replacement for the humeral head. J Bone Joint Surg [Am] 1955;37-A:215-28.

20. Neer CS. Glenohumeral arthroplasty. In: Neer CS II, ed. Shoulder reconstruction. Philadelphia: W.B. Saunders, 1990:143-271.

21. Sperling JW, Cofield RH, Rowland CM. Minimum fifteen-year follow-up of Neer hemiarthroplasty and total shoulder arthroplasty in patients aged fifty years or younger. J Shoulder Elbow Surg 2004;13:604-13.

22. Nystuen CM, Leopold SS, Warme WJ, et al. Cancellous impaction and cortical strut allografting for revision shoulder arthroplasty: a case report. J Shoulder Elbow Surg 2006;15:244-8 